\title{
An unsupervised learning method for reconstructing cell spatial organization with application to the DREAM Single Cell Transcriptomics Challenge [version 1; peer review: 2 not
}

\section{approved]}

\author{
Yang Chen (iD), Disheng Mao², Yuping Zhang², Zhengqing Ouyang (iD) \\ ${ }^{1}$ Department of Biostatistics and Epidemiology, School of Public Health and Health Sciences, University of Massachusetts, Amherst, \\ MA, USA \\ ${ }^{2}$ Department of Statistics, University of Connecticut, Storrs, CT, USA
}

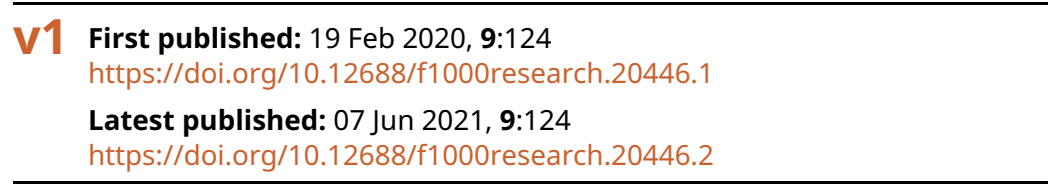

\section{Abstract}

Single cell RNA sequencing (scRNA-seq) data analysis is important for building a global transcription landscape of all cell types in tissues, tracing cell lineages, and reconstructing cell spatial organizations. In this article, we propose an unsupervised learning method to predict spatial positions and gene expression of individual cells in Drosophila embryos using a small number of driver genes. Specifically, we develop a two-stage clustering approach, and compute a probability matrix of the spatial positions of single cells. This method is applied to dataset in the DREAM Single Cell Transcriptomics Challenge. The comparison with the "gold standard" suggests that our method is effective in reconstructing the cell positions and gene expression patterns in spatial tissues.

Keywords

Spatial organization, single cell RNA-seq, Drosophila embryo, clustering, DREAM challenge

\section{Open Peer Review \\ Approval Status $\mathrm{X}$ \\ 1 2 \\ version 2 \\ (revision) \\ 07 Jun 2021 \\ version 1 \\ 19 Feb 2020
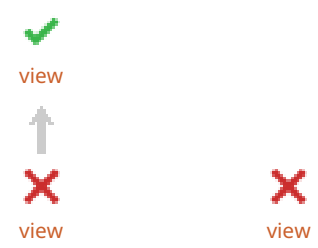 \\ 1. Xianwen Ren ID, Peking University, Beijing, China \\ 2. Mark S. Cembrowski, University of British \\ Columbia, Vancouver, Canada \\ Larissa Kraus, University of British Columbia, \\ Vancouver, Canada}

Any reports and responses or comments on the article can be found at the end of the article. 
Corresponding author: Zhengqing Ouyang (ouyang@schoolph.umass.edu)

Author roles: Chen Y: Data Curation, Formal Analysis, Investigation, Methodology, Software, Validation, Visualization, Writing - Original Draft Preparation; Mao D: Investigation, Methodology; Zhang Y: Funding Acquisition, Investigation, Methodology, Resources,

Supervision, Writing - Review \& Editing; Ouyang Z: Conceptualization, Formal Analysis, Funding Acquisition, Investigation, Methodology, Project Administration, Resources, Supervision, Writing - Review \& Editing

Competing interests: No competing interests were disclosed.

Grant information: This work was partially supported by the Faculty Research Excellence Program Award at UConn (to YZ). The funders had no role in study design, data collection and analysis, decision to publish, or preparation of the manuscript.

Copyright: ( $) 2020$ Chen Y et al. This is an open access article distributed under the terms of the Creative Commons Attribution License, which permits unrestricted use, distribution, and reproduction in any medium, provided the original work is properly cited.

How to cite this article: Chen $Y$, Mao D, Zhang $Y$ and Ouyang $Z$. An unsupervised learning method for reconstructing cell spatial organization with application to the DREAM Single Cell Transcriptomics Challenge [version 1; peer review: 2 not approved] F1000Research 2020, 9:124 https://doi.org/10.12688/f1000research.20446.1

First published: 19 Feb 2020, 9:124 https://doi.org/10.12688/f1000research.20446.1 


\section{Introduction}

The development of single cell RNA sequencing (scRNA-seq) has provided a powerful solution for building a global transcription landscape of all cell types in tissues, finding new cell types, cell lineage tracing, spatial reconstruction, and combining with other omics ${ }^{1-4}$. The single cell is originally made from dissociated tissues without spatial information, and spatial gene expression pattern is unknown. In situ hybridization (ISH) and its variants can detect the spatial information of mRNA transcripts and produce gene expression reference atlas. Using enough marker genes, users can reconstruct the spatial position of single cell RNA-seq data by combing the ISH reference atlas ${ }^{3-7}$. Some works have also combined sequential fluorescence in situ hybridization (seqFISH) and multiplexed error robust fluorescence in situ hybridization (MERFISH) with scRNA-seq data to map cell types to the reference atlas ${ }^{8-10}$.

Recent methods have successfully mapped cells from scRNA-seq data to the spatial positions using dozens of landmark genes ${ }^{3-7}$. Nikos et al. developed a DistMap method for mapping the $\sim 1300$ Drosophila embryo cells into $\sim 3000$ bins in the spatial position using 84 marker genes ${ }^{3}$. Satija ${ }^{4}$ et al. mapped 851 cells of zebrafish embryo into 64 bins in spatial embryo using 47 genes. Kaia et al. computed the correspondence score and mapped 139 cells into a Platynereis dumerilii brain using a set of 98 genes $^{5}$. Andreas et al. reconstructed spatial single enterocytes along the villus axis in 1-D space using 50 bottom and top landmark genes for 1383 cells $^{6}$. Mor et al. proposed novoSpaRc for spatial mapping of the scRNA-seq cells into an existing reference atlas to infer spatial gene expression? ${ }^{7}$. In these methods, the dimension and resolution of the spatial region, as well as the number of marker genes, are key factors to affect the recovery of the spatial position.

The DREAM Single Cell Transcriptomics Challenge aims to develop new algorithms to find embryo spatial pattern. Participating teams are asked to predict the positions in the embryo of 1297 cells using the expression pattern from 60 (sub challenge 1), 40 (sub challenge 2), and 20 (sub challenge 3) driver genes from in situ hybridization data. The challenge is different from the published methods as it endeavors to use less marker genes to infer the spatial locations of cells.

In this article, we introduce an unsupervised learning approach for the three challenges, and validate the results using the "gold standard" method derived from DistMap which uses 84 genes. The paper is organized as follows: Methods, we briefly describe the solutions for all three sub-challenges; Results, we present results of three sub-challenges on the data of the DREAM Single Cell Transcriptomics Challenge; finally, we discuss our results and summarize our work.

\section{Methods}

Dataset

The dataset is from Drosophila embryos. The scRNA-seq dataset is from 1000 handpicked stage 6 fly embryos using Drop-seq ${ }^{11}$. It contains both raw and normalized UMI counts with 1297 cells and 8924 genes per cell. A total of 84 driver genes are used. In situ hybridization expression patterns are from the Berkeley Drosophila Transcription Network Project (BDNTP). The BDNTP reference atlas are binarized. The bin number of one half of the embryo is 3039 . The spatial coordinates of these bins are also specified. The dataset files can be downloaded from the DREAM Single Cell Transcriptomics Challenge after registration with Synapse free of charge (https://www.synapse. org/\#! Synapse:syn16782360).

We directly use the normalized scRNA-seq data, the in situ matrix and the geometry of the embryo. The gene names "E.spl.m5. $H L H$ " and "Blimp.l" are replaced by "E(spl)m5-HLH" and "Blimp-l".

\section{Gene selection}

We use a hierarchical clustering method to select 60,40 , and 20 driver genes from the 84 genes based on the normalized scRNA-seq data.

Based on the belief that the scRNA-seq gene expression pattern is similar to the driver genes' pattern, we propose to select the essential driver genes based on the information provided by scRNA-seq data. Namely, if two genes have high correlation in the scRNA-seq data, we assume the same pattern happens in the in situ matrix. Therefore, we choose only one of them without losing too much of the information. To find the correlated genes, we perform hierarchical clustering on the normalized scRNA-seq data to separate all 84 genes into 60 clusters (with the Euclidean distance and the Mcquitty linkage). The Mcquitty linkage gives more weights for objects in small clusters than those in large clusters in calculating the distance between two clusters. Thus, it is suitable for situations with many small clusters. Since the numbers of clusters are fairly large in the sub-challenge 1 and 2, we opt to use the Mcquitty linkage for distance calculation. In sub-challenge 3 , since the total number of clusters is shrunk to 20 , which is smaller than sub-challenge 1 and 2 , we choose to use the ward linkage in the hierarchical clustering part to obtain largersized clusters from the data. After this step, the gene selection process remains the same as sub-challenge 1 and 2 .

After getting the clusters, we pick the most representative gene of each cluster by calculating the distance between each member gene and the cluster center based on the Euclidean distance and selecting the closest one.

\section{Binarization of scRNA-seq data}

We perform binarization on the normalized scRNA-seq data for the selected genes based on the "binarizeSingleCellData()" function in DistMap (https://github.com/rajewsky-lab/distmap). The details of binarization is as follows: for each quantile threshold, we perform binarization on the scRNA-seq data for each gene. If the gene expression value is larger than the quantile gene expression value, it will be set as 1 , otherwise it is 0 . Then we compute the difference between the correlation matrix of binarized scRNA-seq data and the correlation matrix of in situ matrix based on the root-mean-square error. Last, we select the quantile threshold which has the smallest difference to perform binarization for scRNA-seq data. 
Compute the probability matrix between cells and bins

Given the binarized scRNA-seq data and in situ matrix, we calculate the probability matrix between cells and bins based on the selected driver genes. Here, we assume the selected driver gene number as $\mathrm{n}_{g}$. The probability $p_{i j}$ of a cell $c_{i}(\mathrm{i} \in[1,1297])$ originating from the bin $b_{j}(\mathrm{j} \in[1,3039])$ can be expressed as follows.

$$
p_{i j}=\frac{n_{s}^{i j}}{n_{g}}
$$

$n_{s}^{i j}$ is the number of the same gene expression value ( 0 or 1$)$ in the two binarized vectors of the scRNA-seq data and the in situ matrix for cell $c_{i}$ and bin $b_{j}$.

\section{Select top bins based on the probability matrix}

The probability of a cell originating from a bin is determined by the gene expression in the bin and cell. More genes can improve the prediction of cell position. The bins with the higher probability are possibly the potential cell position. For sub-challenges 1-3, we follow the same process shown in Figure 1. To make the results more stable, we select enough bins (see below) based on the probability values. Then we use clustering to determine a more stable cell position.

To select the potential bins for predicting cell position, we check the distribution of the maximum values in the probability matrix for the bins (Figure 2). Then we use the third quartile of probability values to select the top bins in sub challenge 1 when using 60 driver genes. We use the first quartile of probability values in sub challenge 2 when using 40 driver genes. And we use all bins in sub challenge 3 when using 20 driver genes. If the number of selected bins is 0 , the bin which has the maximum probability will be the predicted position. If the number of selected bins is larger than 100, then only the top 100 bins will be kept based on the probability.
To check the effect of threshold on the prediction results, we test our method under different thresholds as shown in Figure 4 (b)-(d).

\section{Silhouette score to determine the hierarchical clustering number}

For high probability bins, we need to perform clustering to select the cluster which has the maximum sum of probability as cell position. Here, we use hierarchical clustering on the selected bins. The cluster number is determined by the silhouette score, which measures the average distance of a point to other points in its cluster compared to the smallest average distance to other clusters. The silhouette score ranges from 1 to +1 . The higher the silhouette score, the closer the point is closer to its own cluster and the farer it is away from other clusters.

\section{Predict cell positions based on the clustering result}

We use the average silhouette score across all points to select the clustering number. We use NbClust package ${ }^{12}$ to perform hierarchical clustering with the "centroid" method. Based on the silhouette score, we obtain the best clustering number. Then we compare the sums of probabilities of all clusters, and select the cluster which has the maximum sum of probabilities. We use the selected cluster center as a reference point to select 10 nearest bins as the top 10 most possible cell positions.

\section{Performance evaluation}

To evaluate the performance of our method, we use the three performance scores in the DREAM challenge (https://www.synapse.org/\#!Synapse:syn17091286, https://github.com/dream-sctc/ Scoring/blob/master/dream_scoring_clean.R). The first scoring metric is the primary score to estimate the precision of the assignment for the single cells. The second scoring metric is the

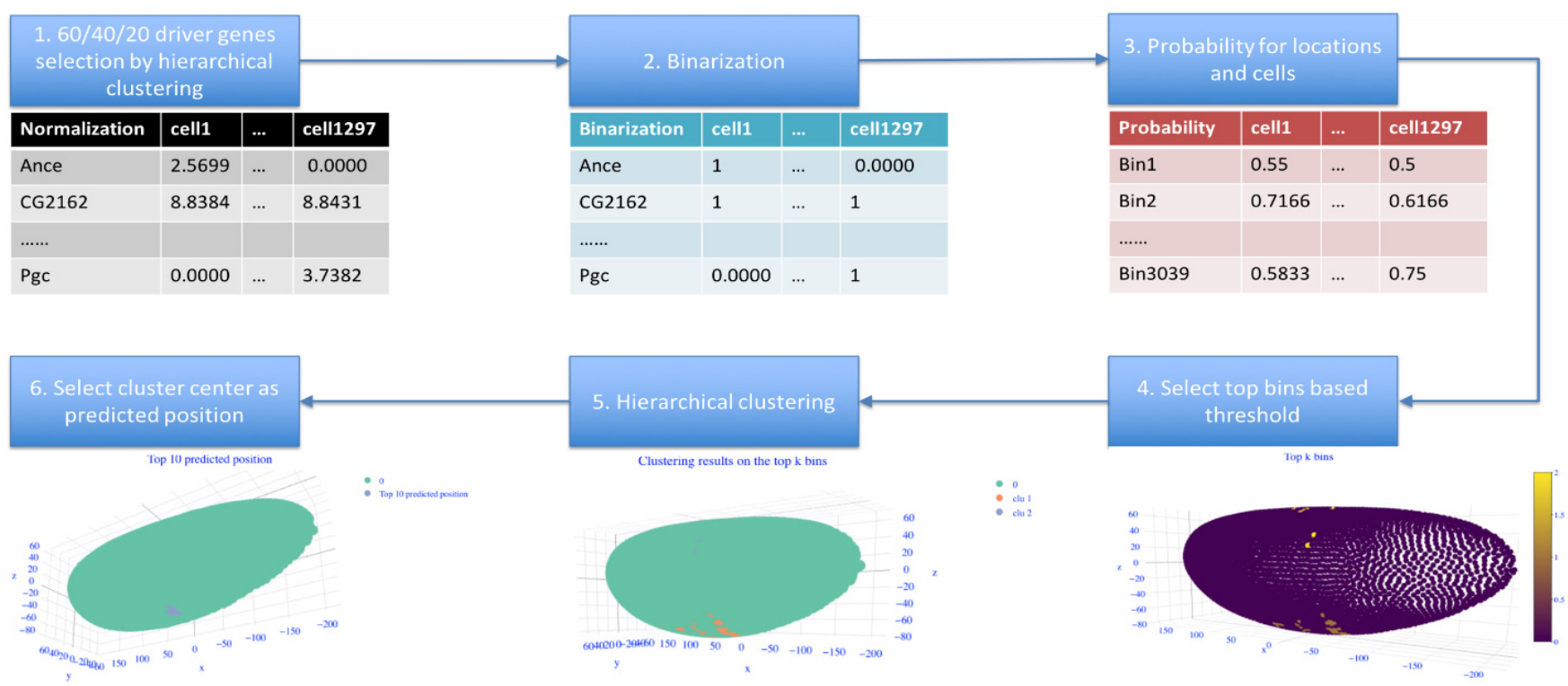

Figure 1. Workflow of our spatial position prediction method for scRNA-seq. 


\section{The distribution of maximum probability value in each bin}

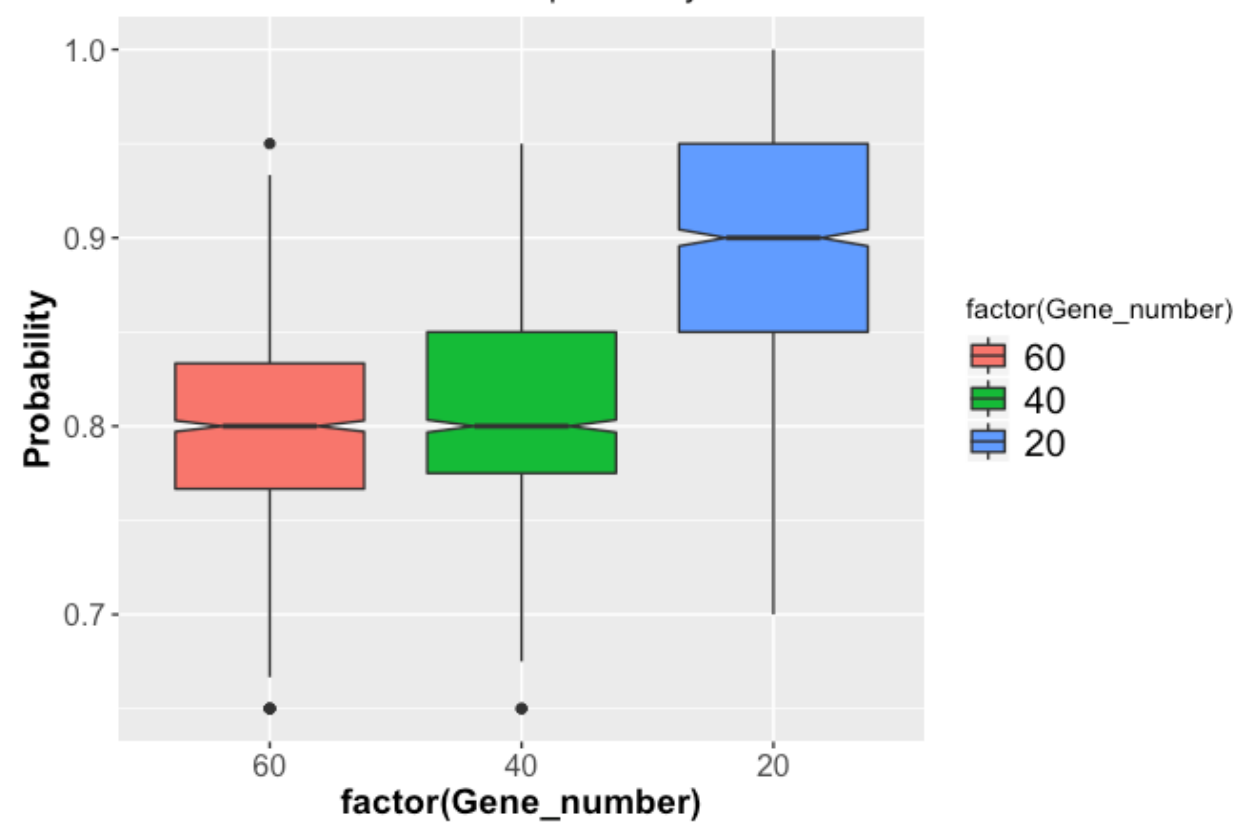

Figure 2. The distribution of the maximum probability values in the probability matrix for the bins for the 60,40 and 20 driver genes scenarios.

average of the relative assignment metrics over all the single cells which is used when the first scores are equal for two methods. The third scoring metric is comparing prediction of gene patterns.

Ambiguous cells: If the predicted top 1 and top 2 positions are the same in the DistMap results, the prediction position will be ambiguous, and the cell will not be computed in the score 1-3. In this challenge, the number of ambiguous cells derived from DistMap are 287.

\section{Results}

\section{Selecting genes}

We calculated the sums of gene expression values in the in situ matrix for all selected driver genes for all 3039 bins. As Figure 3(a) shows, each bin has at least one gene expressed in the in situ matrix. It suggests that our selected driver genes can cover all bins. As the gene number decreases, the frequency of gene expression in each bin decreases. We also compared the overlapped genes in Figure 3(b). Among the 40 driver genes of the sub challenge 2, only one driver gene is not in the selected 60 driver genes of the sub challenge 1. Similarly, only 2 driver genes of the sub challenge 3 are not in the selected 60 driver genes. It suggests our method is consistent in selecting different number of driver genes.

\section{Compare the predicted position and spatial gene expression}

We used the score 1-3 to evaluate our method under the different selected driver genes scenarios. Figure 4(a) shows the scores of our submitted results for the sub-challenge 1,2,
3. The blue bar is the score for the gold standard method using 84 driver genes from DistMap. For score 1, our method is close to the gold standard in sub challenge 1 when using 60 driver genes. The results of our method in sub challenge 2, 3 shows a larger difference. For score 2, our method shows high scores when using 60 and 40 driver genes. Score 2 is the average relative precision for all cells. It suggests that our method is robust for predicting the right position. The score 3 shows a small difference in our method when using 60 and 40 driver genes. Figure 4(b)-(d) shows the consistency of the score 1-3 over a range of thresholds in the different numbers of driver genes scenarios. Hence, Figure 4 shows that our method can obtain a close performance to the gold standard when using 60 driver genes.

As shown in Figure 5, the spatial gene expression prediction accuracy is represented by MCC correlation between the predicted cell position in the in situ matrix and the binarized scRNA-seq data for each driver gene. Score 3 is based on the MCC correlation for each driver gene used in each sub challenge. Corresponding to Figure 4(a), the MCC between the DistMap (84) and our method (60 or 40) for each driver gene in sub challenges 1 and 2 are very close. In sub challenge 3, the MCC of gene "dpn", "erm", "ftz", " $h$ " from our method are much lower than DistMap. It is consistent to the lower score 3 in sub challenge 3 .

\section{Conclusion}

We described our method and its performance using 60, 40 and 20 driver genes by comparing with the gold standard (DistMap results). In sub challenge 1 , our results shows a close 


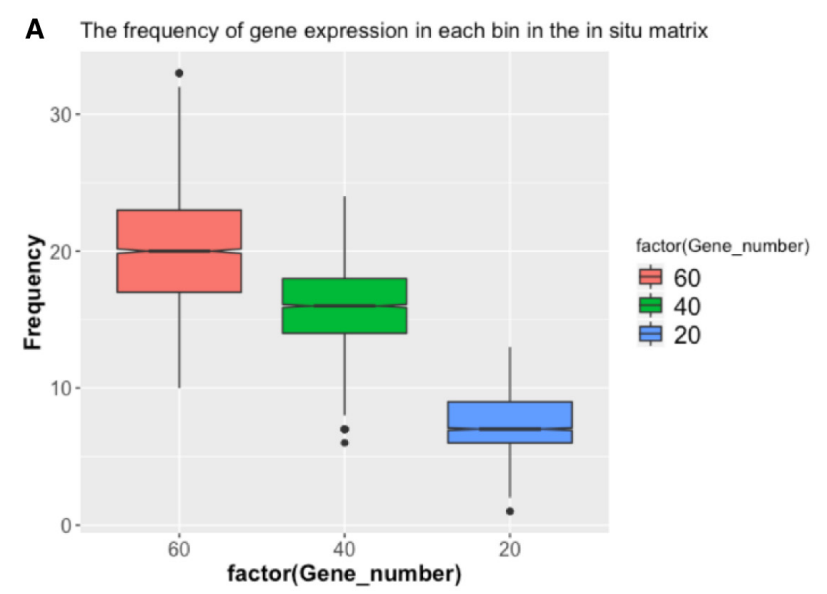

B

\section{The overlaps among selected genes}

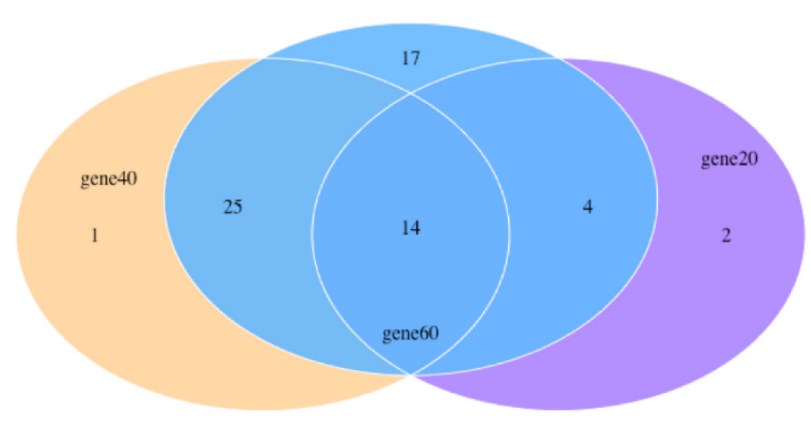

Figure 3. (a) The frequency of gene expression in each bin in the in situ matrix in different selected driver genes scenarios. (b) The overlaps among the selected genes from the three sub challenges.
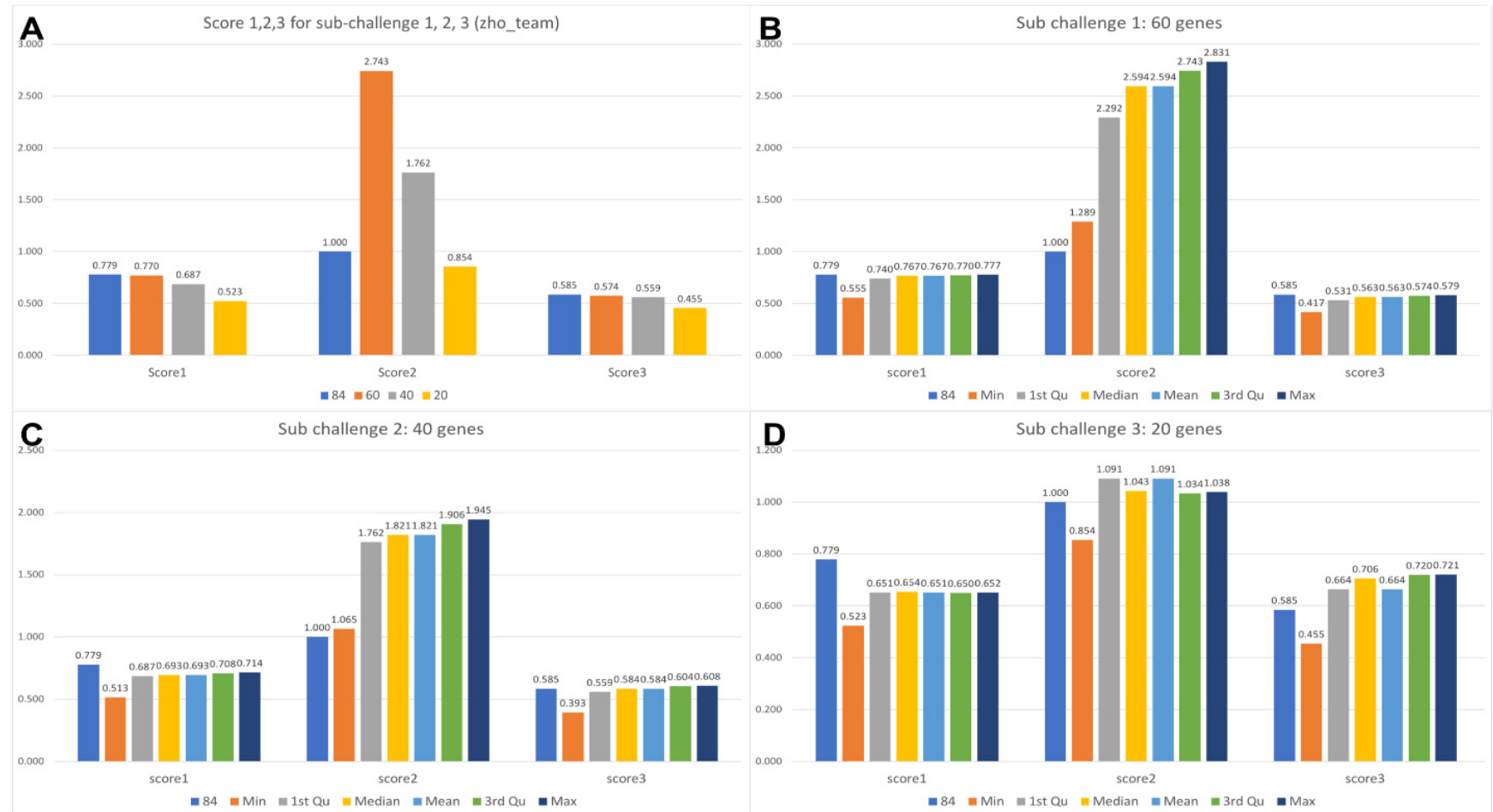

Figure 4. (a) Comparing the score 1, 2, 3 for sub challenge 1 (60 driver genes), 2 (40 driver genes) and 3 (20 driver genes) with the gold standard (84 driver genes). (b-d) Comparing the score 1, 2, 3 using different thresholds for (b) sub challenge 1; (c) sub challenge 2; (d) sub challenge 3. (The numbers are rounded for visualization.)

performance to the gold standard. In sub challenges 2 and 3, when using 40 and 20 driver genes, the score 1 decreases and score 3 is still close to the gold standard. It suggests our method can predict cell positions using 60 genes and predict gene expression patterns using less genes. We tested the threshold for selecting top bins (Figure 4(b)-(d)): the results suggest that our method can achieve even better results when using the maximum threshold for sub challenges 1,2 , and 3 . 
A Compare predicted spatial gene expression accuracy in challenge 1

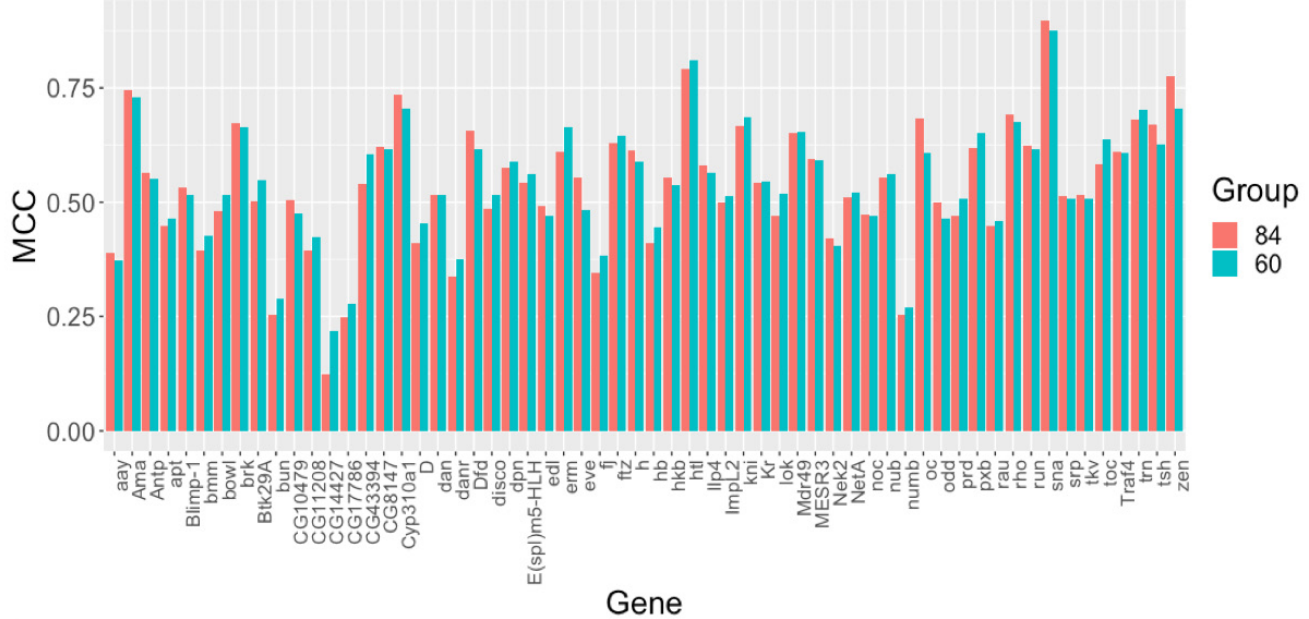

B Compare the predicted spatial gene expression accuracy in challenge 2

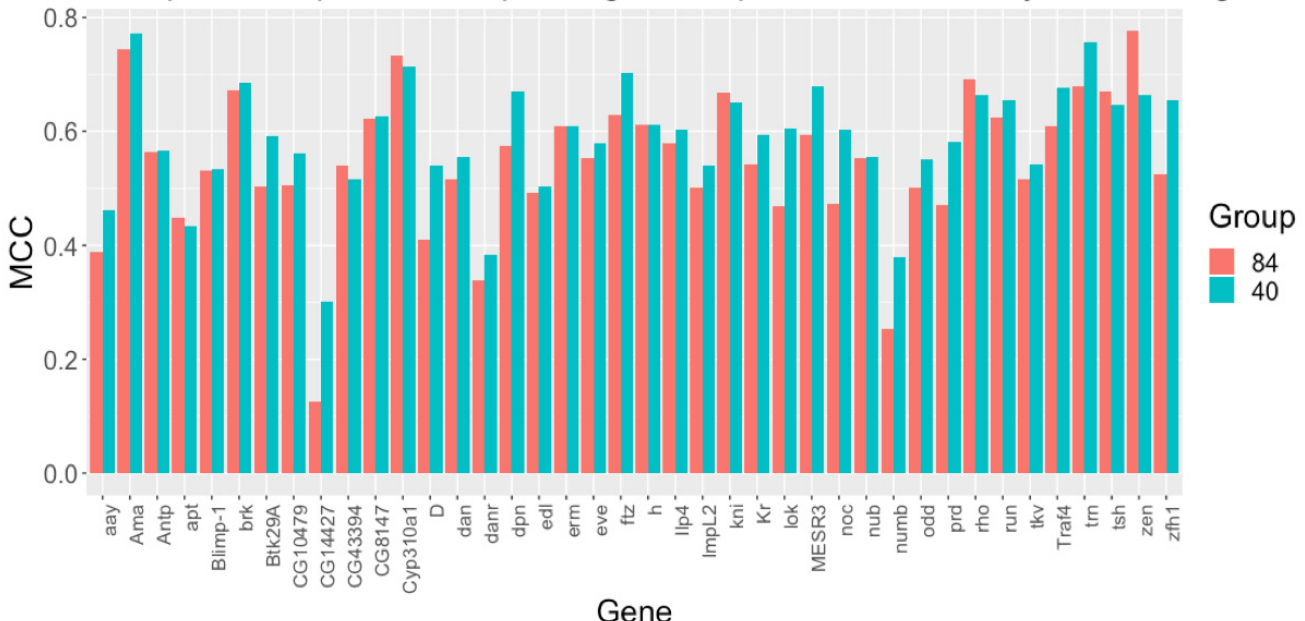

C Compare the predicted spatial gene expression accuracy in challenge 3

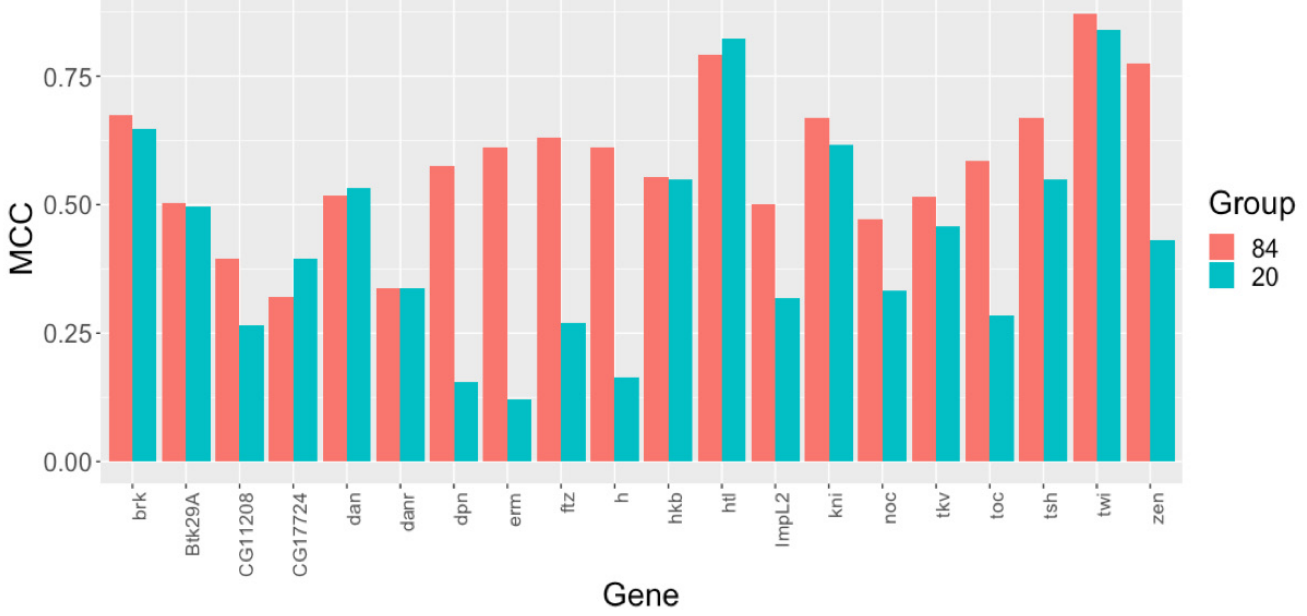

Figure 5. Comparing the spatial gene expression between DistMap (84) with our method using (a) 60 genes in sub challenge 1 ; (b) 40 genes in sub challenge 2; (c) 20 genes in sub challenge 3. 


\section{Data availability}

The dataset associated with the DREAM Single Cell Transcriptomics Challenge is available for registered participants at https://www.synapse.org/\#!Synapse:syn16782360 and https://www.synapse.org/\#!Synapse:syn18632189. Due to sharing protocol of Synapse, users should register in Synapse (free of charge; https://www.synapse.org/) using their email address, and agree to the dataset conditions of use. Once registered, users can download the files.

Synapse: SCTC Challenge zho_team Submission. Code and results underlying this article, https://doi.org/10.7303/syn17056435 $5^{13}$.

\section{Software availability}

Source code implementation for the method presented in this article and used in the DREAM Single Cell Transcriptomics Challenge is available from: https://github.com/ouyang-lab/ SCTC-Challenge-zho_team. Scoring scripts are available from:
https://github.com/dream-sctc/Scoring/blob/master/dream scoring_clean.R.

Archived source code as at time of publication: https://doi. org/10.5281/zenodo. $3592532^{14}$

License: GLP 3.0

Acknowledgements

Data used in this publication were generated by Prof. Dr. Nikolaus Rajewsky, Max Delbrück at the Center for Molecular Medicine, and these results were obtained as part of the DREAM Single Cell Transcriptomics Challenge project through Synapse ID (syn15665609). We thank Pablo Meyer and Jovan Tanevski for providing the documents, code and suggestions for score $1-3$
1. Stuart T, Satija R: Integrative single-cell analysis. Nat Rev Genet. 2019; 20(5): 257-272.

PubMed Abstract | Publisher Full Text

2. Farrell JA, Wang $Y$, Riesenfeld SJ, et al: Single-cell reconstruction of developmental trajectories during zebrafish embryogenesis. Science. 2018; 360(6392): pii: eaar3131.

PubMed Abstract | Publisher Full Text | Free Full Text

3. Karaiskos N, Wahle P, Alles J, et al:: The Drosophila embryo at single-cell transcriptome resolution. Science. 2017; 358(6360): 194-199. PubMed Abstract | Publisher Full Text

4. Satija R, Farrell JA, Gennert D, et al:: Spatial reconstruction of single-cell gene expression data. Nat Biotechnol. 2015; 33(5): 495-502. PubMed Abstract | Publisher Full Text | Free Full Text

5. Achim K, Pettit JB, Saraiva LR, et al:: High-throughput spatial mapping of single-cell RNA-seq data to tissue of origin. Nat Biotechnol. 2015; 33(5): 503-9. PubMed Abstract | Publisher Full Text

6. Moor AE, Harnik $\mathrm{Y}$, Ben-Moshe $\mathrm{S}$, et al.: Spatial reconstruction of single enterocytes uncovers broad zonation along the intestinal villus axis. Cell. 2018; 175(4): 1156-1167.e15.

PubMed Abstract | Publisher Full Text

7. Nitzan M, Karaiskos N, Friedman N, et al:: Charting a tissue from single-cell transcriptomes. bioRxiv. 2018; 456350 .

Publisher Full Text

8. Shah S, Lubeck E, Zhou W, et al.: In situ transcription profiling of single cells reveals spatial organization of cells in the mouse hippocampus. Neuron. 2016; 92(2): 342-357.

PubMed Abstract | Publisher Full Text | Free Full Text

9. Zhu $\mathrm{Q}$, Shah $\mathrm{S}$, Dries $\mathrm{R}$, et al:: Identification of spatially associated subpopulations by combining scRNAseq and sequential fluorescence in situ hybridization data. Nat Biotechnol. 2018; 36: 1183-1190. PubMed Abstract | Publisher Full Text | Free Full Text

10. Moffitt JR, Bambah-Mukku D, Eichhorn SW, et al: Molecular, spatial, and functional single-cell profiling of the hypothalamic preoptic region. Science. 2018; 362(6416): pii: eaau5324.

PubMed Abstract | Publisher Full Text | Free Full Text

11. Macosko EZ, Basu A, Satija R, et al: Highly Parallel Genome-wide Expression Profiling of Individual Cells Using Nanoliter Droplets. Cell. 2015; 161(5): 1202-1214

PubMed Abstract | Publisher Full Text | Free Full Text

12. Charrad M, Ghazzali N, Boiteau V, et al: NbClust: An R Package for Determining the Relevant Number of Clusters in a Data Set. J Stat Softw. 2014; 61(6): 1-36. Publisher Full Text

13. Chen $Y$, Mao $D$, Zhang $Y$, et al:: SCTC Challenge zho_team Submission Synapse [dataset]. 2019. http://www.doi.org/10.7303/syn17056435

14. yancychy: ouyang-lab/SCTC-Challenge-zho_team v0.1 (Version v0.1). Zenodo. 2019.

http://www.doi.org/10.5281/zenodo.3592532 


\section{Open Peer Review}

\section{Current Peer Review Status: $\mathrm{X} X$}

\section{Version 1}

Reviewer Report 23 September 2020

https://doi.org/10.5256/f1000research.22477.r70705

(C) 2020 Cembrowski $\mathbf{M}$ et al. This is an open access peer review report distributed under the terms of the Creative Commons Attribution License, which permits unrestricted use, distribution, and reproduction in any medium, provided the original work is properly cited.

\section{Mark S. Cembrowski}

Dept. of Cellular and Physiological Sciences, University of British Columbia, Vancouver, BC, Canada Larissa Kraus

Department of Cellular and Physiological Sciences, University of British Columbia, Vancouver, BC, Canada

In this manuscript, the authors describe an approach to infer spatial gene expression using scRNA-seq data. The authors aimed to identify spatial expression of cell clusters using 20,40 or 60 marker genes, and compared to the "gold standard" Distmap method using 84 marker genes.

The authors begin to explain the rationale of the manuscript by describing the concept of the DREAM challenge, which aims to introduce novel computational techniques to map spatial gene expression using transcriptomic data. Since the DREAM challenge aims to implement novel methods, it is imperative to introduce and carefully compare common methods with the novel proposed approach, which was underdeveloped by the authors. As an example, the "gold standard" method is mentioned several times, though it is not clear what exactly the "gold standard" is or how well-controlled it is. As another key example, a discussion of any kind is completely missing.

The design of figures for a manuscript should be chosen to clearly emphasize the main points of the data. Here, the figures are difficult to interpret because key information and description is missing (e.g., legends are too brief, axis labels are missing, subplot headings are redundant, figure design is inconsistent). In addition, the design and presentation of the figures seem unmotivated and thereby difficult for the reader to see the importance of any particular data or easily draw conclusions from the results. In addition, consistent colour schemes and designs would help the reader to follow the manuscript and understand the results.

\section{Is the rationale for developing the new method (or application) clearly explained?} Yes

Is the description of the method technically sound? 
Yes

Are sufficient details provided to allow replication of the method development and its use by others?

Partly

If any results are presented, are all the source data underlying the results available to ensure full reproducibility?

Yes

Are the conclusions about the method and its performance adequately supported by the findings presented in the article?

No

Competing Interests: No competing interests were disclosed.

Reviewer Expertise: Transcriptomics

We confirm that we have read this submission and believe that we have an appropriate level of expertise to state that we do not consider it to be of an acceptable scientific standard, for reasons outlined above.

Author Response 19 Jan 2021

Zhengqing Ouyang, University of Massachusetts, Amherst, USA

Point-by-point response to the comments of Reviewer 2

\section{Reviewer 2}

In this manuscript, the authors describe an approach to infer spatial gene expression using scRNA-seq data. The authors aimed to identify spatial expression of cell clusters using 20, 40 or 60 marker genes, and compared to the "gold standard" Distmap method using 84 marker genes.

The authors begin to explain the rationale of the manuscript by describing the concept of the DREAM challenge, which aims to introduce novel computational techniques to map spatial gene expression using transcriptomic data.

Since the DREAM challenge aims to implement novel methods, it is imperative to introduce and carefully compare common methods with the novel proposed approach, which was underdeveloped by the authors. As an example, the "gold standard" method is mentioned several times, though it is not clear what exactly the "gold standard" is or how well-controlled it is. As another key example, a discussion of any kind is completely missing.

We thank the reviewer for the constructive comments. The "gold standard" is generated by DistMap, which is the best available single cell position reference used in the DREAM Challenge. DistMap determines single cell position by searching the maximum similarity for cells and spatial bins using the in situ expression of 84 driver genes in the Berkeley 
Drosophila Transcription Network Project (BDNTP). It is shown that the 84 driver gens are sufficient to uniquely and individually label most of the 1,297 cells (Karaiskos, et al. 2017). Thus, it is used in the DREAM Challenge to assess the performance of methods using smaller numbers $(60,40$, and 20$)$ of driver genes. In the revised manuscript, we changed "gold standard" to "silver standard", as the cell positions generated by DistMap are not experimentally validated. We added the description of the DistMap "silver standard" in the Method section.

We also added more introductions to the common methods for the cell position prediction from single cell transcriptome data in the Introduction section. We described the limitation of current methods and explain the motivation to study how the driver gene selection affecting the prediction of cell spatial position.

The comparisons of our methods (Zho_team) and other top performing methods have been described in the DREAM Single Cell Transcriptomics Challenge consortium paper (Gene selection for optimal prediction of cell position in tissues from single-cell transcriptomics data, Life Sci Alliance. 2020 Nov; 3(11): e202000867). Our manuscript is focused on describing the details of our method used in the DREAM Challenge.

We also expanded the Discussion section of our manuscript. In addition to summarizing our method and results, we also pointed that gene-gene correlation and spatial correlation are useful information for spatial cell position and gene expression prediction.

The design of figures for a manuscript should be chosen to clearly emphasize the main points of the data. Here, the figures are difficult to interpret because key information and description is missing (e.g., legends are too brief, axis labels are missing, subplot headings are redundant, figure design is inconsistent). In addition, the design and presentation of the figures seem unmotivated and thereby difficult for the reader to see the importance of any particular data or easily draw conclusions from the results.

In addition, consistent colour schemes and designs would help the reader to follow the manuscript and understand the results.

We thank the reviewer for the constructive comments. In the revised manuscript, we improved all figures and legends. We enlarged the font sizes for all figures. In Figure 1, we clarified the whole workflow to display the motivation of our method. In Figure 2, we changed it to display the number of bins with high similarity values $(>0.8)$ in the similarity matrix for each cell when the number of driver genes is 60 , 40, or 20 . In Figure 3, we enlarged the font size and added more information in the figure labels. In Figure 4, we enlarged the font size. In figure 5, we changed it to the comparison of the Pearson correlation of predicted gene expression from DistMap (using 84 driver genes) and our method (using 60,40 , or 20 genes).

Competing Interests: No competing interests were disclosed. 
Reviewer Report 02 September 2020

https://doi.org/10.5256/f1000research.22477.r69520

(C) 2020 Ren X. This is an open access peer review report distributed under the terms of the Creative Commons Attribution License, which permits unrestricted use, distribution, and reproduction in any medium, provided the original work is properly cited.

\section{Xianwen Ren}

Biomedical Pioneering Innovation Center, Peking University, Beijing, China

Reconstructing the spatial information of single cells from single-cell RNA-seq data is a pivotal question to further release the revolutionary power of the scRNA-seq technology. Here the authors propose a computational method to infer the spatial positions of single cells of Drosophila embryos based on scRNA-seq data and a reference spatial map based on in situ hybridization of 84 driver genes. While the method is finely tuned for this specific scientific question, some concerns exist.

First, the title is misleading. The authors claimed "an unsupervised learning method". This is not valid because this method uses the reference spatial map of Drosophila embryos. Unless the method only uses the scRNA-seq data, it cannot be claimed "an unsupervised learning method".

Second, the current method has only been evaluated on one dataset. More independent evaluations should be added to demonstrate the generalizing capacity of this method.

Overall, this manuscript addresses an important scientific question. But the method needs more justifications and evidence to demonstrate its novelty and power.

Is the rationale for developing the new method (or application) clearly explained? Yes

Is the description of the method technically sound?

Yes

Are sufficient details provided to allow replication of the method development and its use by others?

Yes

If any results are presented, are all the source data underlying the results available to ensure full reproducibility?

No source data required

Are the conclusions about the method and its performance adequately supported by the findings presented in the article?

Partly 
Competing Interests: No competing interests were disclosed.

Reviewer Expertise: Bioinformatics

I confirm that I have read this submission and believe that I have an appropriate level of expertise to state that I do not consider it to be of an acceptable scientific standard, for reasons outlined above.

Author Response 19 Jan 2021

Zhengqing Ouyang, University of Massachusetts, Amherst, USA

Point-by-point response to the comments of Reviewer 1

\section{Reviewer 1}

Reconstructing the spatial information of single cells from single-cell RNA-seq data is a pivotal question to further release the revolutionary power of the SCRNA-seq technology. Here the authors propose a computational method to infer the spatial positions of single cells of Drosophila embryos based on sCRNA-seq data and a reference spatial map based on in situ hybridization of 84 driver genes. While the method is finely tuned for this specific scientific question, some concerns exist.

1. First, the title is misleading. The authors claimed "an unsupervised learning method". This is not valid because this method uses the reference spatial map of Drosophila embryos. Unless the method only uses the sCRNA-seq data, it cannot be claimed "an unsupervised learning method".

We thank the reviewer for the comments. To clarify, we used unsupervised hierarchical clustering for gene selection. Based on the selected genes, we predicted the spatial position of each individual cell. Unsupervised and supervised gene selection approaches are used by the methods in the DREAM Single Cell Transcriptomics Challenge for predicting cell spatial position. Table S2 of the consortium paper (Gene selection for optimal prediction of cell position in tissues from single-cell transcriptomics data, Life Sci Alliance. 2020 Nov; 3(11): e202000867) listed the top performing methods (included ours) using either unsupervised or supervised gene selection.

2.Second, the current method has only been evaluated on one dataset. More independent evaluations should be added to demonstrate the generalizing capacity of this method.

We thank the reviewer for the constructive comments. The results of our method (Zho_team) and other top performing methods have been systematically and independently evaluated on the Drosophila embryo dataset in the DREAM Single Cell Transcriptomics Challenge (https://www.synapse.org/\#!Synapse:syn15665609/wiki/583234) and in the consortium paper aforementioned.

Our manuscript, collected in the F1000Research DREAM Challenges Gateway (https://f1000research.com/gateways/dreamchallenges/singlecelltranscriptomics), is focused on describing the details of our method used in the DREAM Challenge. To avoid 
ambiguity, in the revised manuscript, we changed the title of our manuscript to "Unsupervised gene selection for predicting cell spatial positions in the Drosophila embryo".

Overall, this manuscript addresses an important scientific question. But the method needs more justifications and evidence to demonstrate its novelty and power.

We thank the reviewer for the comments. Our method is the only one that uses unsupervised hierarchical clustering for gene selection among the top performing methods as described in Table S2 of the DREAM Challenge consortium paper mentioned above. The power has been systematically demonstrated in our manuscript as well as the consortium paper using independent measurements and comprehensive comparisons.

Competing Interests: No competing interests were disclosed.

Reviewer Response 15 Jun 2021

Xianwen Ren, Peking University, Beijing, China

The authors have clarified my concerns.

Competing Interests: NA

The benefits of publishing with F1000Research:

- Your article is published within days, with no editorial bias

- You can publish traditional articles, null/negative results, case reports, data notes and more

- The peer review process is transparent and collaborative

- Your article is indexed in PubMed after passing peer review

- Dedicated customer support at every stage

For pre-submission enquiries, contact research@f1000.com 